\title{
May I Ask Who's Calling? Named Entity Recognition on Call Center Transcripts for Privacy Law Compliance
}

\author{
Micaela Kaplan \\ Brandeis University / Waltham, MA \\ CallMiner Inc / Waltham, MA \\ micaela@brandeis.edu
}

\begin{abstract}
We investigate using Named Entity Recognition on a new type of user-generated text: a call center conversation. These conversations combine problems from spontaneous speech with problems novel to conversational Automated Speech Recognition, including incorrect recognition, alongside other common problems from noisy user-generated text. Using our own corpus with new annotations, training custom contextual string embeddings, and applying a BiLSTM-CRF, we match stateof-the-art results on our novel task.
\end{abstract}

\section{Introduction}

When a call center says "a call may be recorded", they are often collecting a transcript. These transcripts are the output of speech recognition systems, and while they are redacted for Payment Card Industry (PCI) compliance, they often contain other information about the caller such as their name and internal ID number. This data can be helpful for quality assurance and future customer care. New privacy laws, such as the General Data Protection Regulation (GDPR) in the EU, define rules and regulations for everything from how data is collected and stored to the rights of a person to retract their consent to the use of their data (gdp, 2019). In the face of these new laws, it is important to be able to identify non-public personal information and personally identifiable information (NPI/PII) in call transcripts in order to comply with regulations without compromising the data these companies rely on.

We use Named Entity Recognition (NER) to find instances of NPI/PII, remove them from a transcript, and replace them with a tag identifying which type of information was removed. For example, a transcript containing "This is john doe reference number 12345 " would become "This is
[NAME] reference number [NUMBER]". This problem is unique in a call center for a few reasons. Firstly, call transcripts are organic human conversations and present many of the common problems of user-generated data, including false starts, incomplete sentences, and novel words. Secondly, the text provided in a transcript is the output of an Automatic Speech Recognition (ASR) system, which is prone to error as described in Section 3.1. While modern ASR systems are reliable, our input audio is from phone calls, which are usually very lowquality and often contain a lot of background noise. This low-quality audio results in poor ASR, which then outputs sentences that may not be grammatical. This makes it difficult to understand the semantics of the call or to pick up on many of the features that are critical to most NER systems such as context or part of speech. Additionally, production-level call transcripts, or those that are used by Quality Assurance agents and data scientists, are missing capital letters, numeric digits, and accurate punctuation, which are features that are crucial to the classic approaches to NER. Moreover, traditional NER systems use labels for proper nouns, like people's names, but have no way to handle emails, spellings, or addresses, making bootstrapping from pretrained NER models impossible.

In this paper, we apply the current state-of-theart neural architecture for sequence labeling, a BiLSTM-CRF, to our novel call center transcripts in search of NPI and PII as identified by a human. We match state-of-the-art performance for standard datasets on our novel problem by using our model in conjunction with annotated data and custom contextual string embeddings.

\section{Previous Work}

NER became popular in the NLP community at the Message Understanding Conferences (MUCs) 
during the 1990s (Hirschman, 1998). In 2003, the CoNLL2003 shared task focused on language independent NER and popularized feature based systems (Tjong Kim Sang and De Meulder, 2003). The OntoNotes corpus, released in 2006, has also been fundamental to NER research (Hovy et al., 2006).

After CoNLL, the highest performing models were based on a CRF (Lafferty et al., 2001) which requires the manual generation of features. More recently, research has used neural techniques to generate these features. Huang et al. (2015) found great success using Bidirectional Long Short Term Memory models with a CRF layer (BiLSTM-CRF) on both the CoNLL2000 and CoNLL2003 shared task datasets. Ma and Hovy (2016) used a BiLSTMCNN-CRF to do NER on the CoNLL2003 dataset, producing state-of-the-art results. Similarly, Chiu and Nichols (2015) used a BiLSTM-CNN, with features from word embeddings and the lexicon, which produced very similar results. Ghaddar and Langlais (2018) used embeddings for the words and for entity types to create a more robust model. Flair, proposed by Akbik et al. (2018), set the current state of the art by using character based embeddings, and built on this with their pooling approach in 2019 (Akbik et al., 2019). Crossweigh, a framework introduced by Wang et al. (2019), makes use of Flair embeddings to clean mishandled annotations.

In 2006, Sudoh et al. used the word confidence scores from ASR systems as a feature for NER on the recordings of Japanese newspaper articles. In 2018, Ghannay et al. (2018) conducted a similar experiment on French radio and TV audio. Unlike our data, neither of these tasks used spontaneous conversation. Additionally, the audio was probably recording-studio quality, making ASR a reliable task.

\subsection{Conversations are Different: The Twitter Analogy}

All of the previous work discussed was run on datasets primarily comprised of newswire data $(\mathrm{Li}$ et al., 2018). Typically, newswire follows the conventions of normal text, but call center transcripts have none of these conventions guaranteed and often explicitly lack them entirely. This is a problem for the traditional approaches to NER. Our lowquality audio adds to the difficulty of this task.

The closest approximation of this problem in
Speaker 1: Thank you for calling our company how may i help you today.

Speaker 2: Id like to pay my bill.

Figure 1: An example of turns of a conversation, where each person's line in the dialogue represents their turn. This output matches the format of our data described in Section 3.

the previous research is on Twitter data. Tweets, like transcripts, are generated by users and may not follow the grammar, spelling, or formatting rules that newswire is so careful to maintain. In 2011, Liu et al. (2011) used a K-nearest neighbors model combined with a CRF to begin tackling this problem. As part of the 2017 Workshop on Noisy User-generated Text (W-NUT) shared task, Aguilar et al. (2017) obtained a first place ranking using a model that combined a multi-step neural net with a CRF output layer. Akbik et al. (2019) also tested their pooled contextualized string embeddings on this data and found success. We use this previous work on tweets to inform our model creation for the call center space.

\section{Data}

Our dataset consists of 7,953 training, 500 validation, and 534 test samples. Each sample is a complete speaker turn from a call taken by a call center that deals with debt collection. For our purposes, a speaker turn is defined as the complete transcription of one speaker before another speaker starts talking, as illustrated in Figure 1. The training set is a random sample of turns from 4 months of call transcripts from the client, but was manually curated to contain examples of NPI/PII to compensate for its relatively rarity in call center conversation. The transcripts were made using a proprietary speech recognition system, which is set to provide all lowercase transcripts and omits punctuation and numeric digits. We used $\mathrm{spaCy}^{1}$ to convert each turn to a document that starts with a capital letter and ends with a period. This is due to the default configurations of $\mathrm{spaCy}-$ in order to make use of entities, we needed to add in a Sentencizer module, which defaults to this capital letter and period set up.

\subsection{Data Annotation}

We created a schema to annotate the training and validation data for a variety of different categories of NPI/PII as described in Table 1. Initial annota-

\footnotetext{
${ }^{1}$ https://spaCy.io/
} 


\begin{tabular}{|l|l|}
\hline Entity Type & Description \\
\hline NUMBERS & $\begin{array}{l}\text { A sequence of numbers relating } \\
\text { to a customer's information (e.g. } \\
\text { phone numbers or internal ID } \\
\text { number) } \\
\text { NAME } \\
\text { Fomst and last name of a customer } \\
\text { or agent } \\
\text { The name of a company } \\
\text { A complete address, including } \\
\text { city, state, and zip code } \\
\text { Any email address } \\
\text { Language that clarifies the } \\
\text { spelling of a word, (e.g. "a as in } \\
\text { apple") }\end{array}$ \\
SPELLING
\end{tabular}

Table 1: A brief description of our annotation schema.

tion was done with Doccano ${ }^{2}$. These annotations were converted to entities in the text with spaCy. The annotators were trained in NPI/PII recognition, and were instructed to lean towards a greater level of caution in ambiguous cases. This ambiguity was often caused by misrecognitions from the language model in the ASR system being used. With no audio to help the annotator, it wasn't always clear when "I need oak leaves" was supposed to be "Annie Oakley". The reverse problem was also true. "Brilliant and wendy jeff to process the refund" appears to be a full name, but is actually a misrecognition for "Brilliant and when did you want to process the refund". Emails also proved difficult, because misrecognitions made it difficult for annotators to discern exactly what words belonged in the email address. Another difficulty for annotation was that all of our data had been preredacted for PCI compliance, which requires the redaction of number strings relating to credit card numbers, birth dates, and social security numbers. This redaction occurs before any transcript can be released to a client or researcher. To minimize false negatives, PCI redaction frequently redacts numbers that are NPI/PII such as in an internal customer ID number or a phone number. Since the NUMBERS label was intended to catch these NPI/PII related numbers, we used context to include this [redacted] tag as part of a numbers sequence when possible. No steps to clean the transcripts were taken at any point. The naturally occurring noise in our data is critical to our use case and was left for the model to interpret.

Due to limitations with spaCy and the known complexity of nested entities, we opted to allow

\footnotetext{
${ }^{2}$ http://doccano.herokuapp.com/
}

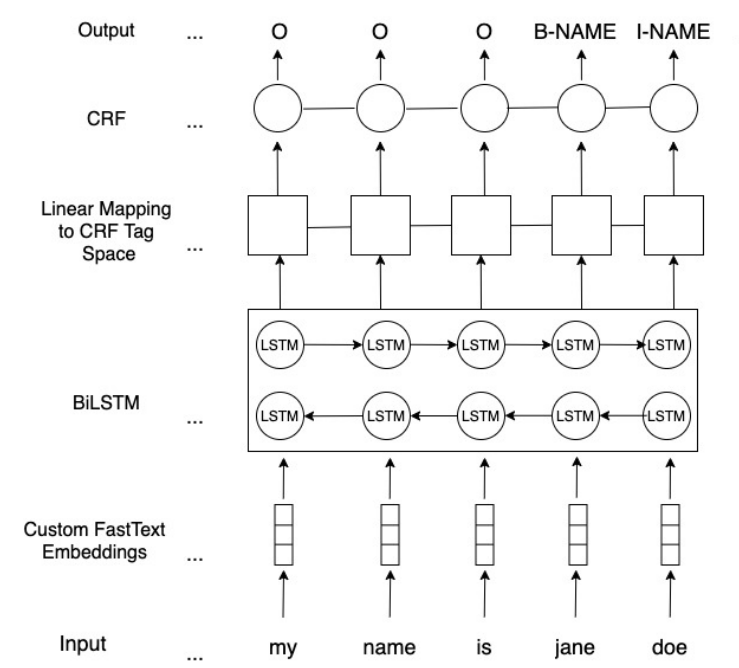

Figure 2: A schematic of our BiLSTM-CRF model. The text of each turn is passed to a word embedding layer which is followed by a BiLSTM layer, and then a linear layer that maps the word BiLSTM output into tag space. Finally, the CRF layer produces an output sequence.

only one annotation per word in our dataset. This means that "c a $t$ as in team at gmail dot com" could only be labeled either as SPELLING[0:6] EMAIL[6:] or as EMAIL[0:] with indices that correspond to the location of the word in the text and are exclusive. This ultimately explains the much lower number of SPELLING entities in the dataset as compared to other entities, because they are often contained a part of EMAIL or ADDRESS. This will influence our analysis in Section 6.

\section{Model Design}

We implemented a standard BiLSTM-CRF model in PyTorch. The basic model implementation is adapted from a GitHub repository ${ }^{3}$. We wrote our own main.py to better allow for our spaCy preprocessing, and we also adapted the code to handle batch processing of data. After this preprocessing, we trained the model with the training set and used the validation set for any model tuning. All reported numbers are on the test set and occur after all tuning is completed. A visualization of our model is found in Figure 2.

\section{Experiments}

\subsection{Basic Hyperparameter Tuning}

We used a grid search algorithm to maximize the performance of the model. The word embedding layer uses FastText embeddings trained on the

\footnotetext{
${ }^{3}$ https://github.com/mtreviso/ linear-chain-crf
} 
client's call transcripts. We find that this helps mitigate the impacts of poor ASR in other aspects of our research, and investigate this further in Sections 5.2 and 5.3. The grid search contained the following parameters: epochs (a sampled distribution between 5 and 50), the size of a dropout layer (between 0 and .5, with .1 intervals of search), the number of hidden layers (between 5 and 20 in increments of 5), and the encoding type used in the output of the CRF (BIO, BILOU, IO). The other hyperparameters in our model were learning rate .001 , batch size 1,30 nodes in each fully connected layer, and the inclusion of bias in each layer. The experiments were run in parallel using Python's multiprocessing package on a virtual machine with 16 CPUs and 128GB of memory. Each experimental configuration ran on a time scale of a few hours, relative to the configurations of the hyperparameters being used.

To better understand the performance of our model on the test set, we broke down the precision, recall, and F1 measurements by entity type. Table 2 shows these results for the best model configuration under the columns labeled "Custom". This model used 46 epochs, a dropout rate of .2, 5 hidden layers, and a BIO encoding.

\subsection{Training Word Embeddings}

While much of the previous research has fine-tuned existing word embeddings, the task of compensating for misrecognition seemed less straightforward than domain adaptation. We lessen the impact of the misrecognitions described in Section 3.1 by understanding that frequent misrecognitions appear in contexts similar to the intended word. For example, "why you're" is often misrecognized as "choir" which would have a totally out of context vector from a pretrained model in this data set. A custom model gives "choir" a vector that is more similar to "why" than to "chorus". Huang and Chen (2020) showed the importance of domain specific word embeddings when using ASR data.

We ran our best performing model configuration with the 300 dimensional GloVe $6 \mathrm{~b}$ word embeddings ${ }^{4}$. Our embeddings, in contrast, are trained on approximately 216 million words, making them substantially smaller than other state-of-the-art embeddings used today. The results from the best epoch of this model (16) are shown in Table 2.

\footnotetext{
${ }^{4}$ https://nlp.stanford.edu/projects/ glove/

${ }^{5}$ Our custom model gets all 0s because many of its pre-
}

\begin{tabular}{|c|cc|cc|cc|}
\hline Entity Type & \multicolumn{2}{|c|}{ Precision } & \multicolumn{2}{c|}{ Recall } & \multicolumn{2}{c|}{ F1 } \\
\hline & Custom & GloVe & Custom & GloVe & Custom & GloVe \\
\cline { 2 - 7 } O & 89.8 & 84.2 & 81.7 & 76.6 & $\mathbf{8 5 . 6}$ & 80.2 \\
NUMBERS & 95.6 & 88.7 & 85.4 & 82.9 & $\mathbf{9 0 . 1}$ & 85.7 \\
NAME & 89.6 & 92.1 & 91.1 & 88.7 & 90.3 & 90.3 \\
COMPANY & 98.8 & 99.5 & 72,9 & 64.3 & $\mathbf{8 3 . 9}$ & 78.1 \\
ADDRESS & 70.6 & .3 & 75.0 & 18.7 & $\mathbf{7 2 . 7}$ & 23 \\
EMAIL $^{5}$ & 0 & 07.1 & 0 & 03.1 & 0 & $\mathbf{0 4 . 4}$ \\
SPELLING & 45.8 & .34 & 52.4 & $40 / 5$ & $\mathbf{4 8 . 9}$ & 37.0 \\
\hline Micro Average & 89.2 & 85.6 & 79.6 & 74.0 & $\mathbf{8 4 . 1}$ & 79.4 \\
\hline
\end{tabular}

Table 2: The performance by entity type of the BiLSTM-CRF model on the held out test set. This table compares the results of our custom embeddings model ("Custom") against the GloVe embeddings ("GloVe").

\subsection{Using Flair}

In our previous experiments, we established the importance of using custom word embeddings to accurately account for the misrecognitions, false starts, and other kinds of noise present in call center conversation transcripts. In this experiment, we test the performance of Flair ${ }^{6}$ and its contextual string embeddings on our data.

We begin by training custom contextual string embeddings for this dataset, based on the findings in our original experiments. For training, we use the same corpus as used in Section 5.1. To do this we follow the tutorial on the Flair GitHub page using their suggested hyperparameter settings as follows: hidden_size: 1024, sequence_length: 250, mini_batch_size: 100, and otherwise use the default parameters. We use the newline to indicate a document change, and list each turn as a separate document to provide consistency with the other experiments conducted in this paper. Given the size of our corpus for word embedding training, we found that our model's validation loss stabilized after epoch 4 . We use the best version of model, as given by Flair, in all of our tests.

We conduct a number of experiments using Flair's SequenceTagger with default parameters and a hidden_size of 256. We adapt the work done by Akbik et al. (2018) and Akbik et al. (2019) to explore the impact of call center data on these stateof-the-art configurations.

Flair uses only the custom trained Flair embeddings.

Flair $_{+}$FastText uses the custom trained Flair embeddings and our custom trained FastText embeddings using Flair's StackedEmbeddings.

Flair $_{\text {mean pooling }}$ uses only the custom trained Flair embeddings within Flair's PooledFlairEmbedding. We use mean pooling due to the results of

\footnotetext{
dicted EMAIL entities were off by a few words. We discuss this more in Section 6.

${ }^{6}$ https://github.com/flairNLP/flair
} 


\begin{tabular}{|c|c|c|c|c|}
\hline Entity & Flair & Flair $_{+}$FastText & Flair $_{\text {mean pooling }}$ & Flair $_{\text {mean pooling + FastText }}$ \\
\hline O & 98.3 & 98.5 & 98.2 & 98.5 \\
NUMBERS & 83.1 & $\mathbf{8 7 . 9}$ & 87.7 & 86.2 \\
COMPANY & $\mathbf{8 1 . 1}$ & 80.7 & 80.7 & 80.3 \\
ADDRESS & 87.5 & $\mathbf{9 4 . 1}$ & 61.5 & $\mathbf{9 4 . 1}$ \\
EMAIL & 58.8 & 50.0 & $\mathbf{7 3 . 3}$ & 66.7 \\
SPELLING & 55.0 & 57.1 & 55.8 & $\mathbf{5 7 . 9}$ \\
Micro Average & 97.5 & 97.7 & 97.3 & 97.7 \\
\hline
\end{tabular}

Table 3: The F1 scores on the test set for each entity type for each Flair embedding experiment.

Akbik et al. (2019) on the WNUT-17 shared task.

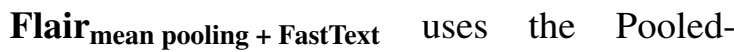
FlairEmbeddings with mean pooling and the custom trained FastText embeddings using Flair's StackedEmbeddings.

These results are shown in Table 3.

\section{Discussion}

Table 2 shows that in all cases except for EMAIL, it is beneficial to use our custom embeddings over GloVe embeddings. We explain this in the next paragraph. The Flair embeddings show a large improvement over the other word embedding varieties however in our circumstance all four varieties of Flair models have nearly identical Micro Average F1s. The best performing Flair models are those that use both the custom contextualized string embeddings and the custom FastText embeddings.

Across all of the models in this paper, EMAIL and SPELLING consistently performed worse than other categories. We believe this is due to the overlap in their occurrences as well as the variability in their appearance. In many cases the custom embeddings model identified parts of an email correctly but attributed certain aspects, like a name, as NAME followed by EMAIL instead of including them together as EMAIL. SPELLING often appears within an EMAIL entity, such as in "c as in cat a $t$ at gmail dot com". Due to the limitations discussed in Section 3, this leads to a limited occurrence of the SPELLING entity in our training data, and many EMAIL and ADDRESS entities that contain examples SPELLING. All models, especially the custom embeddings model, frequently misidentified EMAIL as SPELLING and vice versa. Additionally, our test data contained a number of turns that consisted of only SPELLING on its own, which was poorly represented in train-

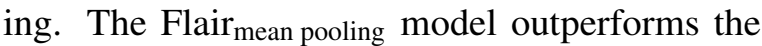
other models in EMAIL by a large margin.

The results shown in Table 3 highlight other interesting notes about our data. The NUMBERS category contains many strings that appear consistently in the text. Not only are there a finite number of NUMBER words in our corpus (those numeric words along with many instances of "[redacted]"), but the NUMBERS of interest in our dataset, such as account numbers, appear in very similar contexts and do not often get misrecognized. The COMPANY entity performs well for a similar reason. When the model was able to identify the company name correctly, it was usually in one of the very common misrecognition forms and in a known context, which furthers our claim that dataset specific embeddings give an important boost over pretrained embeddings. Where the models failed here can likely be attributed to training data. Since the name of the company is a proper noun, it is not in most standard ASR language models, including the one we use. Thus, it is a frequent candidate for misrecognition, because the language model has higher probabilities assigned to grammatically correct phrases that have nothing to do with the name of the company. This leads to high variability in appearance, which means that not every possible version of the company name was present in our training set.

Interesting variability also occurred in ADDRESS entities. With ADDRESS, both models that used Flair and FastText embeddings strongly outperformed the models that used Flair on its own, but standard Flair embeddings strongly outperformed the Pooled Flair embeddings. Neither version of the Flair only model identified addresses in which house numbers or zip codes were shown as "[redacted]" but both models that utilized FastText had no issue with these examples.

\section{Conclusion and Future Work}

By using a BiLSTM-CRF, in conjunction with custom-trained Flair embeddings, we match current state-of-the-art NER performance on our novel call center conversation dataset with unique entity types. We also reinforce the importance of training word embeddings that fully capture the nuances of the data being used for the task. While we cannot release any data for privacy reasons, we have shown that current state-of-the-art techniques successfully carry over to more non-traditional datasets and tasks. In the future, we'd like to assess the contribution of this model with the call transcripts from other industries. Additionally, we'd like to investigate the success of these strategies on other usergenerated conversations, such as chats and emails. 


\section{Acknowledgments}

Thanks to the anonymous reviewers for their invaluable feedback. Thanks to CallMiner Inc. and its research partners for providing all of the data as well as the use cases and funding. Thanks to Jamie Brandon for her help with model architecture design. Thanks to the whole CallMiner research team for their help and support throughout the process.

\section{References}

2019. What is gdpr, the eu's new data protection law?

Gustavo Aguilar, Suraj Maharjan, Adrian Pastor López Monroy, and Thamar Solorio. 2017. A multitask approach for named entity recognition in social media data. Proceedings of the 3rd Workshop on Noisy User-generated Text.

Alan Akbik, Tanja Bergmann, and Roland Vollgraf. 2019. Pooled contextualized embeddings for named entity recognition. In NAACL 2019, 2019 Annual Conference of the North American Chapter of the Association for Computational Linguistics, page 724-728.

Alan Akbik, Duncan Blythe, and Roland Vollgraf. 2018. Contextual string embeddings for sequence labeling. In COLING 2018, 27th International Conference on Computational Linguistics, pages 16381649.

Jason P. C. Chiu and Eric Nichols. 2015. Named entity recognition with bidirectional lstm-cnns.

Abbas Ghaddar and Phillippe Langlais. 2018. Robust lexical features for improved neural network namedentity recognition. In Proceedings of the 27th International Conference on Computational Linguistics, pages 1896-1907, Santa Fe, New Mexico, USA. Association for Computational Linguistics.

Sahar Ghannay, Antoine Caubrière, Yannick Estève, Antoine Laurent, and Emmanuel Morin. 2018. Endto-end named entity extraction from speech.

L Hirschman. 1998. The evolution of evaluation: Lessons from the message understanding conferences. Computer Speech Language, 12(4):281 305.

Eduard Hovy, Mitch Marcus, Martha Palmer, Lance Ramshaw, and Ralph Weischedel. 2006. Ontonotes: the $90 \%$ solution. In Proceedings of the human language technology conference of the NAACL, Companion Volume: Short Papers, pages 57-60.

C. Huang and Y. Chen. 2020. Learning asr-robust contextualized embeddings for spoken language understanding. In ICASSP 2020 - 2020 IEEE International Conference on Acoustics, Speech and Signal Processing (ICASSP), pages 8009-8013.
Zhiheng Huang, Wei Xu, and Kai Yu. 2015. Bidirectional lstm-crf models for sequence tagging.

John Lafferty, Andrew Mccallum, and Fernando Pereira. 2001. Conditional random fields: Probabilistic models for segmenting and labeling sequence data. pages 282-289.

Jing Li, Aixin Sun, Jianglei Han, and Chenliang Li. 2018. A survey on deep learning for named entity recognition.

Xiaohua Liu, Shaodian Zhang, Furu Wei, and Ming Zhou. 2011. Recognizing named entities in tweets. In Proceedings of the 49th Annual Meeting of the Association for Computational Linguistics: Human Language Technologies, pages 359-367, Portland, Oregon, USA. Association for Computational Linguistics.

Xuezhe Ma and Eduard Hovy. 2016. End-to-end sequence labeling via bi-directional lstm-cnns-crf.

Katsuhito Sudoh, Hajime Tsukada, and Hideki Isozaki. 2006. Incorporating speech recognition confidence into discriminative named entity recognition of speech data. In Proceedings of the 21st International Conference on Computational Linguistics and 44th Annual Meeting of the Association for Computational Linguistics, pages 617-624.

Erik F. Tjong Kim Sang and Fien De Meulder. 2003. Introduction to the CoNLL-2003 shared task: Language-independent named entity recognition. In Proceedings of the Seventh Conference on Natural Language Learning at HLT-NAACL 2003, pages 142-147.

Zihan Wang, Jingbo Shang, Liyuan Liu, Lihao Lu, Jiacheng Liu, and Jiawei Han. 2019. CrossWeigh: Training named entity tagger from imperfect annotations. In Proceedings of the 2019 Conference on Empirical Methods in Natural Language Processing and the 9th International Joint Conference on Natural Language Processing (EMNLP-IJCNLP), pages 5154-5163, Hong Kong, China. Association for Computational Linguistics. 